\title{
A Case of Late-Onset Neurofibromatosis Speculated as Type 7
}

\author{
Ideshita $\mathrm{Y}^{1,2}$, Harada $\mathrm{Y}^{1}$, Yasaki $\mathrm{S}^{*_{3}}$, Sugie $\mathrm{M}^{3}$ and Makiyama $\mathrm{H}^{4}$ \\ ${ }^{1}$ Department of Internal Medicine, Shin-yurigaoka General Hospital, Kawasaki, Kanagawa, Japan \\ ${ }^{2}$ Department of Neurology, Higashiyamato Hospital, Higashiyamato, Tokyo, Japan \\ ${ }^{3}$ Department of Neurology, Shin-yurigaoka General Hospital, Kawasaki, Kanagawa, Japan \\ ${ }^{4}$ Department of Gastroenterology, Shin-yurigaoka General Hospital, Kawasaki, Kanagawa, Japan
}

${ }^{*}$ Corresponding author: Yasaki S, Department of Neurology, Shin-yurigaoka General Hospital, Kawasaki, Kanagawa, Japan, E-mail: krgch160@yahoo.co.jp

Citation: Ideshita Y, Harada Y, Yasaki S, Sugie M, Makiyama H (2016) A Case of Late-Onset Neurofibromatosis Speculated as Type 7. J Case Rep Stud 4(5): 503. doi: 10.15744/2348-9820.4.503

Received Date: October 06, 2016 Accepted Date: November 10, 2016 Published Date: November 12, 2016

\begin{abstract}
A 65-year-old woman developed freckling on her skin from axillary to inguinal regions at age 40. At age 55 and 63, she had respectively, a subcutaneous tumor from her right buttock and a left acoustic tumor removed, in both cases, histopathological analyses diagnosed a schwannoma. At age 65, she was admitted to our hospital for a detailed examination of an abdominal mass previously detected by MRI. The patient had no family history of schwannoma, nor presented café-au-lait skin spots. The abdominal mass was diagnosed as a retroperitoneal tumor by enhanced-CT-scan, MRI and FDG-PET/CT. Needle biopsy examinations confirmed a schwannoma diagnosis. Thus, we considered this as a very rare case of late-onset neurofibromatosis speculated as type 7.
\end{abstract}

Keywords: Café-Au-Lait Spot; Late-Onset Form; Neurofibromatosis; Schwannoma

\section{Introduction}

Neurofibromatosis (NF), which was first reported by von Recklinghausen [1], is an autosomal dominant neurocutaneous syndrome [2]. Riccardi proposed a useful NF classification system, which divides NF forms into eight types [3]. Most types of NF present schwannoma or neurofibroma and high incidence of café-au-lait spots. NF type 1 (NF-1, also called von Recklinghausen disease) is the most common neurofibromatosis, occurring in 1 in 3,000 to 4,000 individuals in the United States and worldwide, and the prevalence of NF type 2 (NF-2) is around 1 in 25,000 individuals in the United States or 1 in 33,000 people worldwide [4,5]. An estimated 100,000 Americans have a neurofibromatosis disorder, which occurs in both sexes and in all races and ethnic groups. Most nerve-associated tumors are benign, although occasionally they may become malignant [4]. NF type 3 (NF-3) to type 8 (NF8) are very rare but accurate their incidences are not known.

According to criteria set forth in a National Institute of Health (NIH) consensus conference [6], the patient with NF-1 (classic form) must present two or more of the following clinical features: (1) six or more café-au-lait spots over 5 mm in maximum diameter for prepubertal patients, and over $15 \mathrm{~mm}$ maximum diameter in postpubertal patients, (2) two or more neurofibromas of any type, or one plexiform neurofibroma, (3) axillary or inguinal freckling, (4) optic glioma, (5) two or more Lisch nodules, (6) a distinctive osseous lesion, (7) a first-degree relative with NF-1 by these criteria [1,6]. As for NF-2 (central form), diagnosis requires at least one of these features to be present: (1) bilateral eighth nerve masses, (2) a first-degree relative with NF-2 and either unilateral eighth nerve mass, or two of the following lesions: neurofibroma, meningioma, glioma, schwannoma, or juvenile posterior subcapsular lenticular opacity [6]. NF-3 (mixed form), NF type 4 (variant form), NF type 5 (NF-5: segmental form) and NF type 6 ("spot" form) all show café-au-lait spots [3]. In NF type 7 (NF-7: late-onset form), neurofibromas do not become apparent before the end of the third decade and café-au-lait spots are not observed $[1,3,6]$. NF-8 (unspecified form) has typical features of NF but does not fit into one of the aforementioned categories [3]. NF-7 occurs rarely in late adulthood (from age 30 onward) and is characterized by schwannoma or neurofibroma, skin freckling, but no café-au-lait spot [3]. In this report, we present a first Japanese case of late onset NF speculated as type 7 which is pointed out in the literature.

\section{Case Report}

A 65-year-old woman developed freckling on her skin from axillary to inguinal regions at age 40. At age 55, a small subcutaneous tumor was removed from her right buttock, and histopathologically diagnosed as a schwannoma. At age 63, she experienced leftear hearing loss, a left acoustic tumor was identified and removed, and histopathological analyses indicated another schwannoma. 
At age 65, she was admitted to our hospital for a detailed examination of an abdominal mass, 20 mm in maximum diameter, previously detected by MRI. The patient had no family history of schwannoma, and presented freckling without café-au-lait spots (Figure 1A). No Lisch nodules were found upon ophthalmological examination. She reported left-ear hearing loss, but no other neurological abnormalities were observed. Enhanced-CT-scan revealed a retroperitoneal tumor, 20 mm in maximum diameter, positioned just behind the duodenum (Figure 1B). Abdominal lymph node enlargement was not present. FDG-PET/CT showed a high FDG-uptake lesion in the same location, confirming CT scan findings. No abnormal FDG-uptake lesions suggesting a malignant tumor were observed (Figure 1C). Needle biopsy of the retroperitoneal tumor was next performed. Histopathological analyses of the removed tissue revealed the presence of Antoni type A tissue, consisting in fascicles of elongated, bipolar cells, typical of schwannoma (Figure 1D).

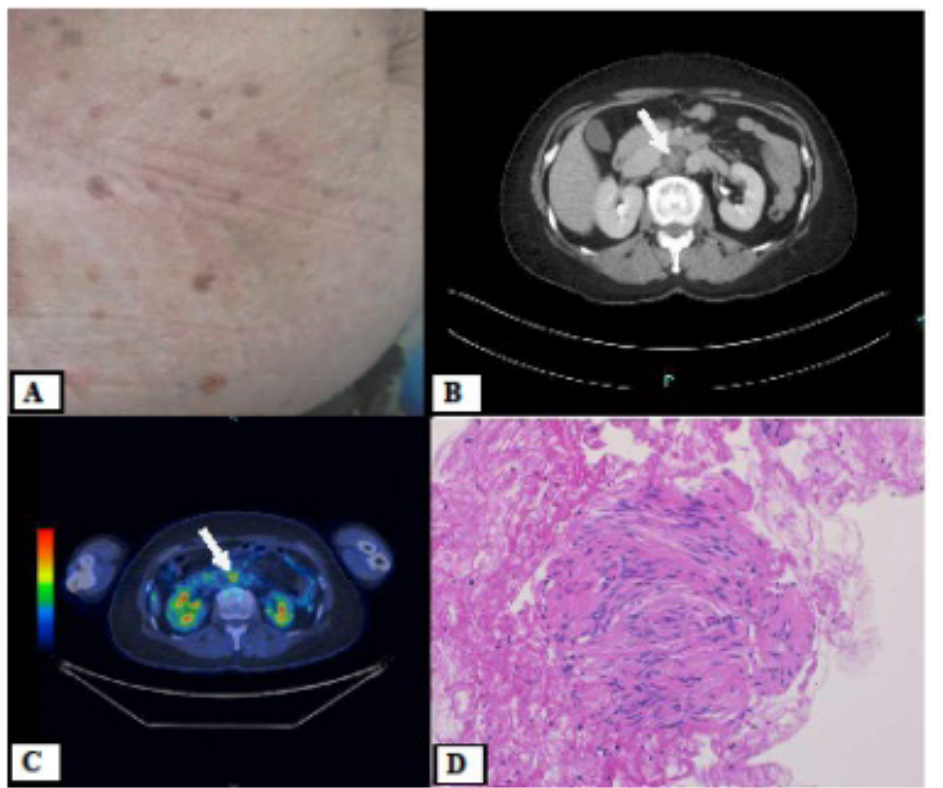

Figure 1: (A) Skin freckling from axillary to inguinal regions. Café-au-lait spot are not observed; (B) Enhanced-CT-scan reveals the presence of a retroperitoneal mass just behind the duodenum (arrowhead). Abdominal lymph node enlargement is not presents; (C) FDGPET/CT shows a high FDG-uptake lesion at the level of the enhanced CT-scan-identified mass (arrowhead), indicating a retroperitoneal tumor. No abnormal FDG-uptake lesions suggesting a malignant tumor are observed; (D) Hematoxylin-eosin stained tissue biopsied by needle from the retroperitoneal tumor. Cells present a narrow, elongated, and wavy shape, with tapered ends interspersed with collagen fibers, typical of schwannoma

Based on these findings, we propose a diagnosis of NF-7, which is a late-onset NF form associated with freckling and schwannomas but not with café-au-lait spots.

\section{Discussion}

NF is inherited in an autosomal dominant manner [2]. NF-1 and NF-2 genes are respectively localized on chromosomes 17q11 and 22q12 [1,2,7]. The NF-1-associated gene product is neurofibromin, which influences cell proliferation and differentiation, whereas the NF-2-associated gene product is merlin (or schwannomin) [7]. Recently the neurofibromatoses are considered as a group of three genetically distinct disorders that cause tumors to grow in the nervous system. The disorders have classified as NF-1, NF-2, and a type that was once considered to be a variation of NF-2 but is now called schwannomatosis. The reason of occurrence of these tumors is not completely known. These mutations have the genes identified as NF1, NF2 and SMARCB1/ INI1 [4]. On the other hand, sporadic cases can also occur [1]. The criteria set forth in a NIH consensus conference is consisted of clinical aspects including family history of NF [6], As to a diagnostic criteria for NF-2, modifications to NIH consensus diagnostic criteria for NF-2 have been suggested to enable initial assessment for diagnosing people who present without bilateral vestibular schwannomas as having NF-2, particularly people with a negative family history of NF-2 by Baser et al. in 2002 [8,9]. In the modified criteria for NF-2, following new two conditions are added to NIH consensus diagnostic criteria such as: (1) unilateral vestibular schwannoma and any two of meningioma, schwannoma, glioma, neurofibroma, posterior subcapsular or lenticular opacities, or (2) multiple meningiomas and unilateral vestibular schwannoma or any two of schwannoma, glioma, neurofibroma or cataract. These modified clinical diagnostic criteria for NF-2 have been found to improve sensitivity substantially without affecting specificity [8,9]. According to this modified criteria, there may be the possibility of NF2 in our case who has unilateral vestibular schwannoma and two of other schwannomas. But we considered that there is a fundamental discussion whether NF-2 can be diagnosed without family history of NF-2 because this is an inherited disease in an autosomal dominant manner although only approximately $50 \%$ of individuals with NF-2 have an affected parent [8]. The diagnosis of NF-2 is based on clinical criteria and the NIH consensus diagnostic criteria are still used to diagnose the case of NF-2 worldwide [6-8]. The mutational analysis of $\mathrm{NF}$ is not required to make a diagnosis of NF in all criteria for NF [1-3,6,8,9]. Our patient of this case report did not want to take 
a mutational analysis but had no family history of NF-2. And the average age of onset of NF-2 is 18 to 24 years. Almost all affected individuals of NF-2 develop bilateral vestibular schwannomas by age 30 years and individuals older than age 30 years who have a unilateral vestibular schwannoma are at very low risk of developing NF-2 [4,8-10]. But our patient of this case report had unilateral vestibular schwannoma at age of 63 . Therefore we considered that the possibility of diagnosis of NF-2 according to the modified criteria by Baser et al. in 2002 cannot be denied completely without genetic analysis but the possibility is very low from the view of clinical aspects in our patient.

Because our patient developed axillary and inguinal freckling at age 40 and schwannomas in the left acoustic nerve, right buttock, and retroperitoneal space, but presented no family history of NF nor café-au-lait spots, we speculated this a case of late-onset form of NF (NF-7) according to NIH consensus diagnostic criteria [6].

In 1999, Drews et al. reported one case of NF-7 [2], and Yildirim et al. reported another case of NF-7 in 2004 [11]. These two cases showed no family history of schwannoma, nor café-au-lait spots. The 49-year-old woman reported by Drews et al. presented malignant schwannoma in the left rib. The 68-year-old man reported by Yildirim et al. had cranial meningioma. In Japan, a 57-year-old woman with a late-onset NF type was reported to have developed neurofibromas on her right upper limb at age 30 but not café-au-lait spots, but was diagnosed with a deep-seated segmental NF (NF-5) form in 2000 [12]. And a 59-year-old Japanese man with a late-onset NF type revealing NF1 mutation was reported in 2015 [13]. He had developed neurofibromas on the trunk and bilateral extremities without café-au-lait spots after the age of 40 . He was a rare case of NF-1 revealing a late-onset NF with mutation of NF1 gene. Table 1 presents clinical features of previously reported NF-7 cases and Japanese late-onset NF cases which descriptions of clinical features are precise.

\begin{tabular}{|c|c|c|c|c|c|}
\hline Case & 1 & 2 & 3 & 4 & 5 \\
\hline Diagnosis & NF-7 & NF-7 & $\begin{array}{l}\text { late-onset NF } \\
\quad(N F-5)\end{array}$ & late-onset NF (NF-1) & $\begin{array}{c}\text { late-onset NF } \\
\text { (speculated as NF-7) }\end{array}$ \\
\hline $\begin{array}{c}\text { Age at diagnosis } \\
\text { (y.o.) }\end{array}$ & 49 & 68 & 57 & 59 & 65 \\
\hline Gender & female & male & female & male & female \\
\hline $\begin{array}{c}\text { Family history of } \\
\text { NF }\end{array}$ & none & none & none & none & none \\
\hline $\begin{array}{c}\text { Tumor } \\
\text { manifestation }\end{array}$ & $\begin{array}{l}\text { neurofibromas of the } \\
\text { right rib, pelvis, right } \\
\text { femur malignant } \\
\text { schwannoma of the } \\
\text { right rib, liver (meta- } \\
\text { stasis) uterine myoma } \\
\text { (benign) }\end{array}$ & $\begin{array}{l}\text { neurofibromas of } \\
\text { the abdomen } \\
\text { meningioma in the } \\
\text { anterior fossa (view } \\
\text { of cranial CT) }\end{array}$ & $\begin{array}{l}\text { neurofibromas of } \\
\text { the right upper } \\
\text { limb at age } 30\end{array}$ & $\begin{array}{l}\text { neurofibromas of the } \\
\text { trunk and bilateral } \\
\text { extremities }\end{array}$ & $\begin{array}{l}\text { schwannomas of the left } \\
\text { acoustic nerve, right buttock } \\
\text { and retroperitoneal space } \\
\text { no malignant tumors } \\
\text { tumor markers were normal }\end{array}$ \\
\hline No café-au-lait spots & None & None & None & None & None \\
\hline Freckling & None & None & none & $\begin{array}{l}\text { freckling on the bilateral } \\
\text { upper back and left lower } \\
\text { back in his } 20 \text { s }\end{array}$ & $\begin{array}{l}\text { freckling on the skin from } \\
\text { axillary to inguinal regions } \\
\text { at age } 40\end{array}$ \\
\hline Lisch nodules & None & None & None & None & None \\
\hline Genetic analysis & not described & not described & not described & mutation of NF1 gene & not examined \\
\hline Therapy & $\begin{array}{l}\text { surgical removal and } \\
\text { chemotherapy }\end{array}$ & surgical removal & surgical removal & not described & surgical removal \\
\hline Patient status & $\begin{array}{l}\text { dead of disseminated } \\
\text { tumor disease }\end{array}$ & Alive & $\begin{array}{l}\text { dead of fluminant } \\
\text { hepatitis }\end{array}$ & Alive & Alive \\
\hline Source year & Drews et al. 1999 & Yildrim et al. 2004 & Ogose et al. 2000 & Uchiyama et al. 2015 & This case 2016 \\
\hline \# of reference & 2 & 11 & 12 & 13 & \\
\hline
\end{tabular}

NF: neurofibromatosis; NF-1: neurofibromatosis type 1; NF-5: neurofibromatosis type 5; NF-7: neurofibromatosis type 7 Table 1: Clinical features of previously reported NF-7 cases

Hence, this report is the first to describe a Japanese case of late-onset NF speculated as NF-7 based on the NIH consensus diagnostic criteria. And we considered that this raises the discussion whether NF-2 can be diagnosed without family history of NF-2 because this is a genetic disorder.

\section{Conclusion}

NF-7 is a very rare type of neurofibromas which occurs rarely in late adulthood and is characterized by schwannoma or neurofibroma, skin freckling, but no café-au-lait spot. In this report, we present a first Japanese case of late-onset NF speculated as NF-7 which is pointed out in the literature. But, according to the modified criteria for NF-2 by Baser et al. (2002), the possibility of diagnosis of NF-2 cannot be denied completely without genetic analysis. 


\section{References}

1. Lin AN, Carter DM (1992) Hereditary cutaneous disorders In: Dermatology ( ${ }^{\text {rd }}$ Edn), WB Saunders Company, Philadelphia, United States.

2. Drews G, Emmrich P, Hauss J, Spiegel HU (1999) Malignant schwannoma and late-onset form of neurofibromatosis (NF-VII type) in a patient with skeletal manifestations. Langenbecks Arch Surg 384: 441-4.

3. Riccardi VM (1982) Neurofibromatosis: clinical heterogeneity. Curr Probl Cancer 7: 1-34.

4. Neurofibromatosis Fact Sheet (2016) National Institute of Neurological Disorders and Stroke (NINDS), USA.

5. Genetics Home Reference (2016) Neurofibromatosis Type 2, USA.

6. Neurofibromatosis (1988) Conference statement. National Institutes of Health Consensus Development Conference. Arch Neurol 45: 575-8.

7. David Perkin G, Miller DC, Lane RJ, Patel MC, Hochberg FH (2011) Neurofibromatosis (von Recklinghausen's disease) In: Atlas of Clinical Neurology (3 ${ }^{\text {rd }}$ Edn), Elsevier Saunders, Philadelphia, United States.

8. Evans DG (2011) Neurofibromatosis 2 In: GeneReviews, Seattle, WA; University of Washington, USA.

9. Baser ME, Friedman JM, Wallace AJ, Ramsden RT, Joe H, et al. (2002) Evaluation of clinical diagnostic criteria for neurofibromatosis 2. Neurology 59: 1759-65.

10. Evans DG, Ramsden RT, Gokhale C, Bowers N, Huson SM, et al. (2007) Should NF2 mutation screening be undertaken in patients with an apparently isolated vestibular schwannoma? Clin Genet 71:354-8.

11. Yildirim M, Kesici D, Baysal V, Candir Ö, Akkaya A (2004) Late-onset neurofibromatosis (NF-7): a case report. Gazi Med J 15: 107-10.

12. Ogose A, Hotta T, Imaizumi S, Saito H, Homma T, et al. (2000) Deep-seated segmental neurofibromatosis without café au lait spots. Skeletal Radiol 29: 543-7.

13. Uchiyama M, Sakai N, Maeda T, Tsuboi R, Mitsuhashi Y (2015) Late-onset neurofibromatosis revealing NF1 mutation. J Dermatol 42: 104-5.

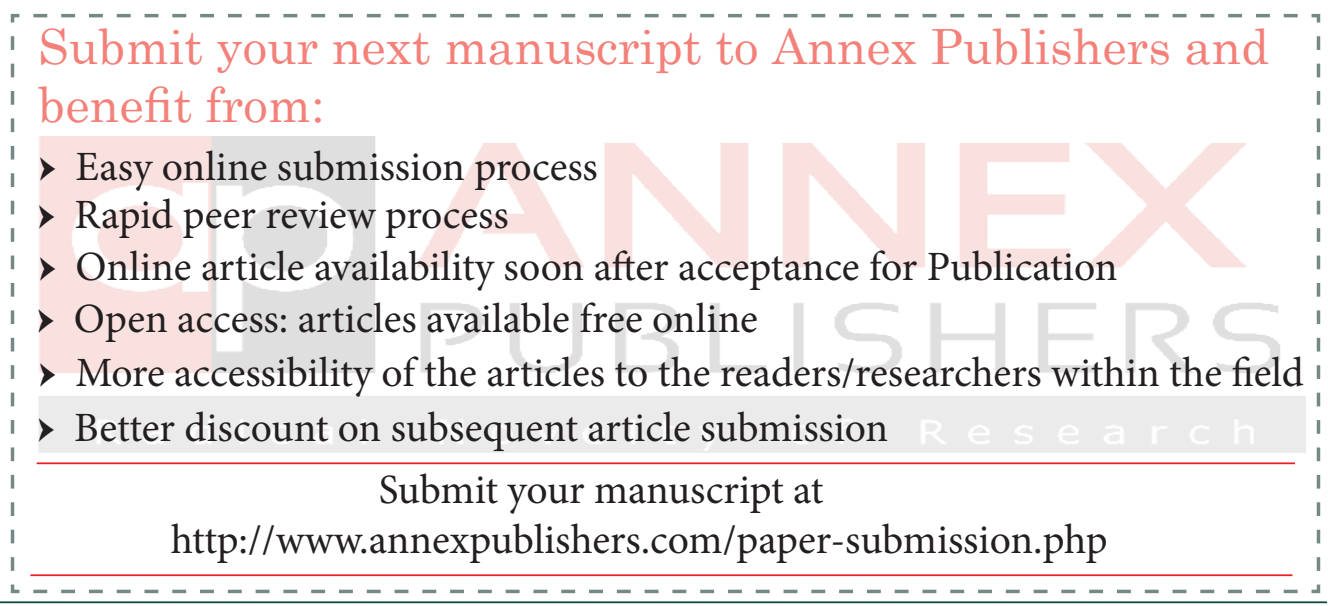

